\title{
Ratios of peripheral blood mononuclear cells to IncRNA steroid receptor RNA activator as new indicators of metabolic syndrome
}

\author{
Liqin Zhao', Shiyi Liang', Chijian $\mathrm{Li}^{2}$, Jianye $\mathrm{Yu}^{2}$, Lihao Zou${ }^{2}$, Weicheng $X \mathrm{v}^{2}$, Ge Qian², Ying Zhang', \\ Yuxiang Huang ${ }^{3 *}$, Yongqiang $L^{3 *}$ \\ ${ }^{1}$ Center of Health Management, The Third Affiliated Hospital of Southern Medical University, Guangzhou, China \\ ${ }^{2}$ Department of Nephrology, Institute of Nephrology and Urology, The Third Affiliated Hospital of Southern Medical University, \\ Guangzhou, China \\ ${ }^{3}$ Department of General Medical, The Third Affiliated Hospital of Southern Medical University, Guangzhou, China \\ *These authors contributed equally to this work.
}

\begin{abstract}
Introduction: Metabolic syndrome (MetS) is a clinical syndrome with several characteristics. Steroid receptor RNA activator (SRA) is a long non-coding RNA (lncRNA), which can increase the expression of steroid receptor-dependent gene. This study aimed to explore the changes in metabolic parameters and the predictive value of the peripheral blood mononuclear cells (PBMCs) to SRA ratios as new indicators in subjects with and without MetS in southern China.

Material and methods: There were 81 participants (39 with MetS and 42 without MetS) in this cross-sectional study. The expression of lncRNAs in PBMCs was evaluated by quantitative reverse transcription polymerase chain reaction (qRT-PCR). The risks of SRA and PBMCs to SRA ratios contributing to the presence of MetS were estimated by univariate and multivariate logistic regression models. The area under the receiver (AUC) operating characteristic curve was employed to evaluate diagnostic accuracy.

Results: MetS was positively correlated with cortisol, interleukin 6 (IL-6), white blood cell to SRA ratio (WTSR), lymphocyte to SRA ratio (LTSR), monocyte to SRA ratio (MTSR), and PBMC to SRA ratio (PTSR). A receiver operating characteristic (ROC) curve analysis was performed to assess the value of LTSR (OR: 0.722; $p<0.001$ ) for predicting MetS. The area under the curve yielded a cut-off value of 0.483 , with a sensitivity of $76.9 \%$ and a specificity of $71.4 \%(p<0.001)$.

Conclusion: In summary, SRA in PBMCs may be an important biomarker of stress reaction and may play a role in vulnerability to MetS. Also, the lymphocyte to SRA ratio demonstrated high accuracy in the diagnosis of MetS. (Endokrynol Pol 2022; 73 (1): 81-86)

Key words: IncRNA; steroid receptor RNA activator; peripheral blood mononuclear cells; metabolic syndrome; biomarkers
\end{abstract}

\section{Introduction}

Metabolic syndrome (MetS) is a combination of several characteristics, including abdominal obesity, raised blood pressure, high triglycerides (TG), low high-density lipoprotein cholesterol (HDL-C), and hyperglycaemia, resulting in increased risk of type 2 diabetes mellitus (T2DM), cardiovascular diseases (CVD), fatty livers, and cancers [1]. Five risk factors with different critical cut-off values are used to identify individuals with the MetS: waist circumference, circulating levels of triglycerides, HDL-C, fasting glucose, and blood pressure [2]. In China, the age-standardized prevalence of MetS was $9.8 \%$ in men and $17.8 \%$ in women in 2001, and the figures have increased to $31.0 \%$ in men and $36.8 \%$ in women in 2010 [3]. Among various risk factors of MetS, chronic stress has emerged as a contributor to the development of MetS. Psychological stress can affect health through complex interactions among neuroendocrine responses and energy homoeostasis [4]. Glucocorticoid (GC) (cortisol in human beings) is the critical matter responding to stress, which can cause central obesity, hypertension, hyperlipidaemia, and glucose intolerance. GC action is mediated by glucocorticoid receptor (GR), a nuclear receptor that regulates physiological events through activation or repression of target genes involved in inflammation, gluconeogenesis, and adipocyte differentiation [5].

Long non-coding RNA (lncRNA), a non-coding RNA with more than 200 nucleotides in length, can modify chromatin structures by interacting with polycomb repressive complex 2, enhance or reduce gene transcription by recruiting transcription factors, and regulate miRNA processing in the cell nucleus. In the cytoplasm, 
lncRNAs can mediate mRNA translation, increase or decrease mRNA stability, and act as miRNA sponges that can repress miRNAs [6]. LncRNAs have been found to be involved in various physiological and pathological cellular activities, such as adipogenesis, inflammation, cell differentiation, and tumourigenesis, via genomic expression modulation, epigenetic modification, and post-transcriptional regulation in cis or in trans by interacting with chromatins, proteins, and RNAs in the nucleus or cytoplasm. LncRNAs can participate in the pathogenesis of various human diseases, including metabolic diseases [7]. In an Indian study, the expression levels of lncRNAs HOTAIR, PANDA, and growth arrest-specific transcript 5 (GAS5) in PBMC in T2DM patients were significantly increased and positively correlated with the expression levels of inflammatory factors: tumour necrosis factor alpha (TNF- $\alpha$ ), IL6, and MCP1. It indicates that there are abnormal expressions of various lncRNAs in the PBMC of diabetic patients, which are closely related to inflammatory factors [8]. Among the inferring lncRNAs mentioned above, GAS5 can competitively bind glucocorticoid response elements (GREs) near the promoter of the target gene that GR specifically binds, thereby inhibiting the transcription of GC-GR-GRE-dependent target genes and changing the downstream effects of GC [9]. SRA can induce the expression of steroid receptor-dependent gene, acting as a scaffold to regulate the expression of stress-related genes. SRA can co-activate GR. More and more studies have shown that SRA plays a key role in lots of diseases such as obesity, cardiomyopathy, and tumourigenesis [10].

Although lncRNAs have received widespread attention as potential and powerful biomarkers in the pathogenesis and progression of many diseases, few studies have investigated the expressions of SRA and GAS5, which can regulate GR in the MetS population. It has been suggested that the gene expression signature in PBMCs may provide an indicator of gene activation changes as a differential response to stress in humans [11]. In this study, we analysed the association between MetS and expression of SRA and GAS5 in PBMCs, which may unveil a new target for the prevention and treatment of stress-related disorders including MetS.

\section{Material and methods}

\section{Subjects}

This study was conducted among the same recruitments in our present study as described in this article [12]. Patients with acute or chronic infections or immunological diseases, obvious liver and kidney dysfunction, severe heart diseases, pregnancy, mental illness or drug abuse, gastrointestinal diseases (such as chronic gastrointestinal disorders, diarrhoea, biliary tract infection and enteritis), and serious diseases of the blood or the endocrine systems, were excluded from the study. A structured questionnaire was used to collect information on demographic data, environmental exposure, and medical histories. Current cigarette smokers were defined as those who smoked $\geq 1$ cigarette/day. Alcohol use was defined as intake of wine/beer/cider/spirits $\geq 1$ time per week. Physical activity was defined as walking or riding $15 \mathrm{~min} /$ day or doing sports or physical exercise $>2 \mathrm{~h} /$ week, or lifting or carrying heavy objects at work daily [13]. This study was approved by the hospital ethical committee, and informed consent was obtained from each participant.

\section{Anthropometric measurement}

Anthropometric parameters including weight, height, waist circumference (WC), hip circumference (HC), and blood pressure were obtained using standard measurement. Body mass index (BMI) was calculated by dividing the weight $(\mathrm{kg})$ by the squared value of height in metres.

\section{Blood samples collection and RNA extraction}

Following an overnight fast, a 5-mL venous blood sample from each subject was collected using EDTA anticoagulant tubes and processed within three hours. A 3-mL sample was immediately centrifuged to retrieve plasma. PBMCs were isolated from $2 \mathrm{~mL}$ whole blood by Ficoll-Hypaque density gradient centrifugation. Immediately afterwards, total RNA was extracted from PBMCs by standard protocol of Trizol reagent (Invitrogen, New York, USA). The purity of RNA was determined using a BioPhotometer plus Eppendorf nucleic acid protein Analyzer (Hamburg, Germany), and the integrity was evaluated using agarose gel electrophoresis stained with ethidium bromide. All RNA used had OD260/ OD280 ratio $>1.8$, and electrophoresis showed that the integrity was acceptable. The plasma and RNA were then stored at $-80^{\circ} \mathrm{C}$ until assayed.

\section{Biochemical analysis}

Fasting plasma glucose (FPG), total cholesterol (TC), triglycerides (TG), and HDLC were measured using standard laboratory methods (Hitachi autoanalyzer 7060; Hitachi, Tokyo, Japan). Low-density lipoprotein cholesterol (LDLC) was calculated using the Friedewald formula. Fasting insulin (FINS) and interleukin 6 (IL-6) was calculated by high-pressure liquid chromatography method (Tosoh Corporation, Tokyo, Japan). Plasma cortisols were measured by commercial radio immunoassays using with accounter (XH-6020; North Institute of BioTech, Beijing, China). Plasma TNF- $\alpha$ concentrations were evaluated by enzyme-linked immunosorbent assay using a microplate reader (STAT FAX 2100; Awareness, Palm City, FL, USA). The intra-assay and inter-assay coefficient of variation were $<5.5 \%$ and $<10.0 \%$ for these assays, respectively. The degree of IR was determined using the HOMA-IR, which was calculated using the following formula: [fasting insulin (mIU/L) * fasting glucose $(\mathrm{mmol} / \mathrm{L})] / 22.5$.

\section{Quantitative real-time PCR}

Quantification was performed with a two-step reaction process: reverse transcription (RT) and quantitative real-time PCR (qPCR). Each RT reaction consisted of $1 \mu \mathrm{g}$ RNA, $2.0 \mu \mathrm{L}$ of $10 \mathrm{mM}$ dNTP (promega), $0.5 \mu \mathrm{L}$ of RNase inhibitor (promega), $0.5 \mu \mathrm{L}$ of universal primer (Qiagen), $0.5 \mu \mathrm{L}$ of lncRNA-specific primer, $4 \mu \mathrm{L}$ of $5 \mathrm{x}$ buffer, $0.5 \mu \mathrm{L}$ of (Moloney Murine Leukemia Virus) Reverse Transcriptase (promega), and $12.0 \mu \mathrm{L}$ of nuclease-free water in a total volume of $20 \mu \mathrm{L}$. Using U6 as the internal reference of lncRNAs, the primers of GAS5 and SRA were designed and synthesized by Shanghai Sangon Biotechnology Co., Ltd. (Shanghai, China). Reactions were performed in an ABI PRISM® 7500 Sequence Detection System (Applied Biosystems, Foster City, USA) for $60 \mathrm{~min}$ at $42^{\circ} \mathrm{C}$, followed by heat inactivation of RT for $10 \mathrm{~min}$ at $85^{\circ} \mathrm{C}$. qPCR was performed with $20 \mu \mathrm{L}$ PCR reaction mixture that included $5 \mu \mathrm{L}$ of cDNA, $10 \mu \mathrm{L}$ of 2x SYBR Green qPCR SuperMix (Invitrogen), $0.5 \mu \mathrm{L}$ of forward primer (Qiagen), $0.5 \mu \mathrm{L}$ of reverse primer, and $4 \mu \mathrm{L}$ of nuclease-free 
water. Reactions were incubated in a 384-well optical plate at $95^{\circ} \mathrm{C}$ for $5 \mathrm{~min}$, followed by 40 cycles of $95^{\circ} \mathrm{C}$ for $15 \mathrm{sec}$ and $60^{\circ} \mathrm{C}$ for 32 sec. The specific generation of expected PCR product was confirmed by automated melting curve analysis. All samples were performed in-triplicate (cDNA from the same PCR reaction but in separate wells). The expression levels of lncRNAs were calculated using the 2- $\Delta \Delta \mathrm{Ct}$ method [ $\Delta \mathrm{Ct}=$ mean $\mathrm{Ct}$ (lncRNA of interest)- mean $\mathrm{Ct}(\mathrm{U} 6)$, $\Delta \Delta \mathrm{Ct}=\Delta \mathrm{Ct}$ of lncRNA of interest in samples to be tested $-\Delta \mathrm{Ct}$ of U6 in reference samples. The up-stream primer sequences were as follows: GAS5 forward primer 5'- GCACACAGGCATTAGACAGA -3'; GAS5 reverse primer 5'-CCATACCCAAGCAAGTCATC; SRA forward primer 5' - GGCCAGTCTCTCTGTTCCAA -3', SRA reverse primer CTTCACCTCTTTCAGCCACTT.

\section{Statistical analysis}

Normality of data distribution was assessed using the KolmogorovSmirnov test. The independent samples test, chi-square test, and nonparametric test were used to compare differences of demographic and clinical parameters between two groups. Spearman's correlation coefficient was used to test the correlation between lncRNA markers, inflammation cells to lncRNAs ratios, and cortisol and other clinical variables. The odds ratios (ORs) and their 95\% confidence intervals (CIs) were calculated to assess the risk of lncRNAs and inflammation cells to lncRNAs ratios contributed to the presence of MetS using both univariate and binary logistic regression models with or without adjustment for covariates. ROC analysis was used to assess the biomarker potential of each lncRNA and inflammation cell to lncRNA ratios for MetS, and the area under the curve (AUC) was used as diagnostic index. The diagnostic performances of the lncRNA and ratio panels were further evaluated using the predicted probability of being diagnosed with MetS as a surrogate marker to construct ROC curve. A p value of less than 0.05 was considered statistically significant. The reported $p$ values were two-tailed in all calculations. All statistical analyses were performed using SPSS 25.0 .

\section{Results}

\section{Basic characteristics of the study subjects}

The demographic and clinical characteristics of the study participants were described in our present work [12].

\section{Comparison of lncRNAs levels and stress hormones between two groups}

The expression levels of GAS5 and SRA in PBMCs and plasma levels of stress hormones including cortisol, IL-6, and TNF- $\alpha$ are listed in Table 1. The expression

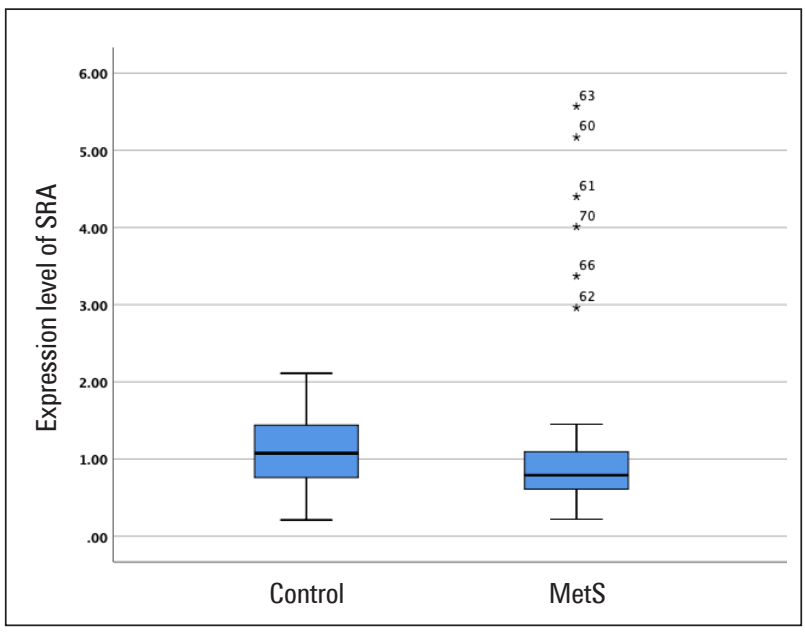

Figure 1. Comparison of steroid receptor RNA activator (SRA) expression in peripheral blood mononuclear cells (PBMCs) between patients with metabolic syndrome (MetS) and control groups. The expression level of SRA in MetS group and control group were $0.79 \pm 0.52$ and $1.08 \pm 0.69$, respectively (median \pm interquartile range), $p$ value was 0.036 , but there were no significant differences of long non-coding RNAs (lncRNA) growth arrest-specific transcript 5 (GAS5) between two groups

level of SRA in MetS patients was significantly lower than that in control individuals $(\mathrm{p}=0.036)$ (Fig. 1), but there was no significance shown in GAS5 ( $p=0.936)$. Levels of plasma cortisol and IL-6 in the MetS group was significantly higher than that in the control group ( $p=0.003$ and $p=0.003$, respectively); however, there were no significant differences of plasma TNF- $\alpha$ levels in the two groups $(\mathrm{p}=0.872)$.

\section{Risk of SRA and the inflammatory cell to SRA ratios for MetS}

Logistic regression revealed that inflammatory cells (including total WBC, LC, MC, and PBMCs) to SRA ratios were positively associated with the presence of MetS ( $\mathrm{p}<0.01)$ (Tab. 2). These associations were also confirmed in binary logistic regression analysis after adjustment for age and gender, and further for physical activity. With an increase of inflammatory cell

Table 1. Long non-coding RNAs (LncRNAs) and hormonal characteristics in the two compared groups

\begin{tabular}{lccc}
\hline Variable & MetS $(\mathbf{n}=\mathbf{3 9})$ & Control $(\mathbf{n}=\mathbf{4 2})$ & p value \\
\hline GAS5 & $1.16 \pm 1.43$ & $1.18 \pm 1.26$ & $0.936^{* *}$ \\
\hline SRA & $0.79 \pm 0.52$ & $1.08 \pm 0.69$ & $0.036^{* *}$ \\
\hline Cortisol $[\mathrm{ng} / \mathrm{mL}]$ & $455.98 \pm 93.80$ & $393.87 \pm 86.34$ & $0.003^{*}$ \\
\hline IL-6 $[\mathrm{pg} / \mathrm{mL}]$ & $3.32 \pm 3.70$ & $2.04 \pm 1.45$ & $0.006^{* *}$ \\
\hline TNF- $\alpha[\mathrm{pg} / \mathrm{mL}]$ & $5.87 \pm 2.03$ & $5.30 \pm 1.97$ & $0.872^{*}$ \\
\hline
\end{tabular}

${ }^{*} \mathrm{p}$ value calculated with independent samples $\mathrm{T}$ test, and the data are expressed as mean $\pm \mathrm{SEM} ;{ }^{* *} \mathrm{p}$ value calculated with nonparametric test, and the data are expressed as median \pm interquartile range; MetS — metabolic syndrome; GAS5 — growth arrest-specific transcript 5; SRA — steroid receptor RNA activator; IL-6 — interleukin 6; TNF- $\alpha$ - tumour necrosis factor alpha 
Table 2. The risk of steroid receptor RNA activator (SRA) and the inflammatory cell to SRA ratios for metabolic syndrome (MetS)

\begin{tabular}{lcc}
\hline Models & OR $(95 \%$ Cl) & p value \\
\hline SRA & & \\
\hline Univariate model & $1.185(0.754,1.863)$ & $0.461^{*}$ \\
\hline Binary model 1 & $1.166(0.699,1.947)$ & $0.557^{* *}$ \\
\hline Binary model 2 & $1.172(0.703,1.954)$ & $0.543^{* * *}$ \\
\hline WTSR & & \\
\hline Univariate model & $1.103(1.018,1.194)$ & $0.016^{*}$ \\
\hline Binary model 1 & $1.125(1.053,1.202)$ & $0.001^{* *}$ \\
\hline Binary model 2 & $1.111(1.025,1.204)$ & $0.011^{* *}$ \\
\hline LTSR & & \\
\hline Univariate model & $1.529(1.13,2.069)$ & $0.006^{*}$ \\
\hline Binary model 1 & $1.527(1.132,2.059)$ & $0.006^{* *}$ \\
\hline Binary model 2 & $1.526(1.13,2.059)$ & $0.006^{* * *}$ \\
\hline MTSR & & \\
\hline Univariate model & $3.200(1.121,9.136)$ & $0.030^{*}$ \\
\hline Binary model 1 & $4.376(1.245,15.384)$ & $0.021^{* *}$ \\
\hline Binary model 2 & $4.343(1.23,15.333)$ & $0.022^{* * *}$ \\
\hline PTSR & & $<0.001^{*}$ \\
\hline Univariate model & $15.336(4.788,49.125)$ & $<0.001^{* *}$ \\
\hline Binary model 1 & $67.392(8.905,509.995)$ & $<.001^{* * *}$ \\
\hline Binary model 2 & $85.432(9.761,747.76)$ & \\
\hline
\end{tabular}

This table shows the logistic regression analysis for the risk of SRA and the inflammatory cells to SRA ratios for MetS. * $p$ value calculated without adjustment; **p value adjusted for age, gender; ${ }^{* * *} \mathrm{p}$ value further adjusted for physical activity based on model 1; $\mathrm{OR}$ - odds ratio; $\mathrm{Cl}$ - confidence interval; WTSR — white blood cell to SRA ratio; LTSR — lymphocyte to SRA ratio; MTSR — monocyte to SRA; PTSR — PBMCs to SRA ratio

to SRA ratios, there were 1.103 (95\% CI: 1.018-1.194, $\mathrm{p}=0.016), 1.529$ (95\% CI: 1.13-2.069, $\mathrm{p}=0.006), 3.200$ (95\% CI: 1.121-9.136, $\mathrm{p}=0.030)$, and 15.336 (95\% CI: 4.788-49.125, $\mathrm{p}<0.001)$-fold greater risk of MetS, respectively. However, the expression level of SRA showed no significant risk of MetS, also after adjustment for age and gender, and further for physical activity. Taken together, inflammatory cell to SRA ratios were all independent risk factors for MetS, especially the ratio of PBMC (consisting of LC and MC) to SRA.

\section{The diagnostic accuracy of $S R A$, the ratios of inflammatory cells to SRA, cortisol, and IL-6 for MetS}

The diagnostic accuracy of SRA, the ratios of inflammatory cells to SRA, cortisol, and IL-6 for MetS was measured by AUC. The accuracy of SRA was $0.635(95 \%$ CI $0.508-0.762, \mathrm{p}=0.036$ ). The sensitivity and specificity were $71.4 \%$ and $69.2 \%$, respectively (Fig. 2). The accuracy of ratios of inflammatory cells to SRA, including the lymphocyte count to SRA ratio (LT1), monocyte count to SRA ratio (MT1), and PBMC to SRA ratio (PT1) were 0.722 (95\% CI: 0.603-0.841, p < 0.001), 0.664 (95\% CI: $0.539-0.789, \mathrm{p}=0.011)$, and 0.718 (95\% CI: 0.598-0.838, $\mathrm{p}<0.001)$, respectively. The sensitivity and specificity of LT1 were $76.9 \%$ and $71.4 \%$, respectively (Fig. 3). The sensitivity and specificity of MT1 were $66.7 \%$ and $76.2 \%$, respectively. The sensitivity and specificity of PT1 were $79.5 \%$ and $66.7 \%$, respectively (Fig. 4). These results revealed that the accuracy of ratios of inflammatory cells to SRA were more valuable than SRA. Among the sensitivity and specificity of ratios of inflammatory cells to SRA, the sensitivity of PT1 and the specificity of MT1 were higher. We also analysed the diagnostic accuracy of cortisol and IL-6 for MetS. The accuracy of cortisol was 0.71 (95\% CI: 0.596-0.824, p = 0.001). The sensitivity and specificity were $79.5 \%$ and $59.5 \%$, respectively. The accuracy of IL-6 was 0.676 (95\% CI: 0.554-0.797, $\mathrm{p}=0.007)$. The sensitivity and specificity were $79.5 \%$ and $59.5 \%$, respectively.

\section{Discussion}

The present study identified the relationship of expression levels of two stress-related lncRNAs in PBMCs and the PBMCs to SRA ratios between MetS patients and healthy controls in an occupational sample in Guangzhou. The expression level of SRA was negatively associated with MetS presence, and it was a significant predictor for MetS. Also, we found that the expression levels of cortisol and IL-6 in MetS were significantly higher compared with healthy controls. Our study also revealed that SRA in PBMCs were potential markers for diagnosing MetS, and the lymphocytes count to SRA ratio was identified to have the greatest accuracy for the diagnosis of MetS. Cortisol has the highest diagnostic sensitivity for MetS among these factors. The main limitation of our study was the number of participants.

GR has two splice variants: GR $\alpha$ and GR $\beta$. GR $\alpha$ is the classical receptor, which is bound with GCs to regulate gene expression, and GR $\beta$ is served as an inhibitor of $\mathrm{GR} \alpha$, which can induce GC resistance [14]. Another study found that the expression level of GR $\alpha$ in myoblast under basal conditions was positively associated with levels of insulin resistance, BMI, percentage body fat, and blood pressure, which may be involved in the pathogenesis of the MetS [15]. But in another study, GR $\alpha$ mRNA was negatively correlated with BMI and triglycerides, and reduced in obesity subjects in the abdominal subcutaneous and omental depots, which is in contrast with the two former observations[16]. The role of GR $\alpha$ in metabolism remains unclear.

As a nuclear receptor coregulator of steroid receptors (including GR), lncRNA SRA can enhance the transcriptional activity of steroid receptors genes. lncRNA SRA 


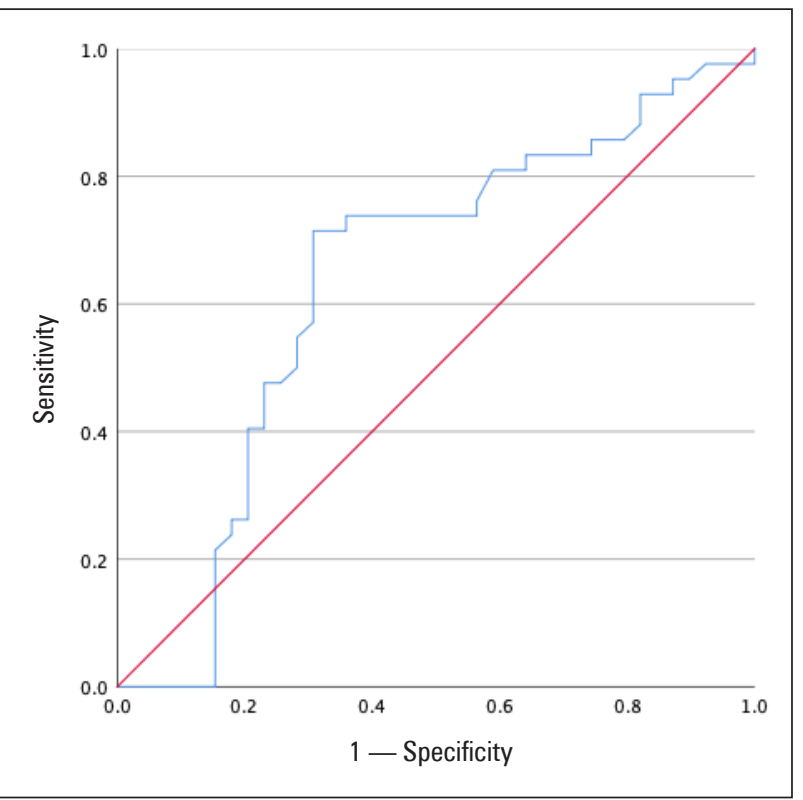

Figure 2. Receiver operating characteristic (ROC) curve analysis of steroid receptor RNA activator (SRA) for metabolic syndrome (MetS) diagnosis. The accuracy of SRA was 0.635 (95\% CI: $0.508-0.762), p=0.036$. The sensitivity and specificity were $71.4 \%$ and $69.2 \%$, respectively. AUC - area under the curve

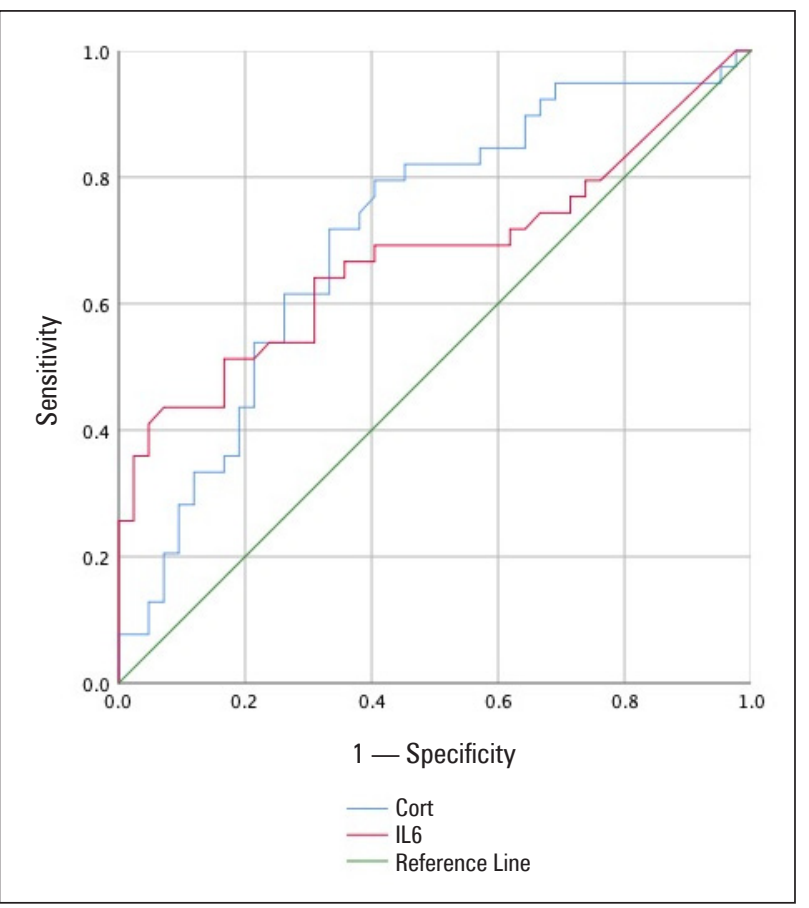

Figure 4. Receiver operating characteristic (ROC) curve analysis of cortisol and interleukin 6 (IL-6) for metabolic syndrome (MetS) diagnosis. The accuracy of cortisol and IL-6 were 0.722 (95\% CI: $0.603-0.841, p<0.001), 0.664$ (95\% CI: 0.539-0.789, $p=0.011$ ), and 0.718 (95\% CI: $0.598-0.838, p<0.001)$, respectively. The sensitivity and specificity of lymphocyte to SRA ratio (LTSR) were $76.9 \%$ and $71.4 \%$, respectively. The sensitivity and specificity of monocyte to SRA ratio (MTSR) were $66.7 \%$ and $76.2 \%$, respectively. Diagonal segments are produced by ties

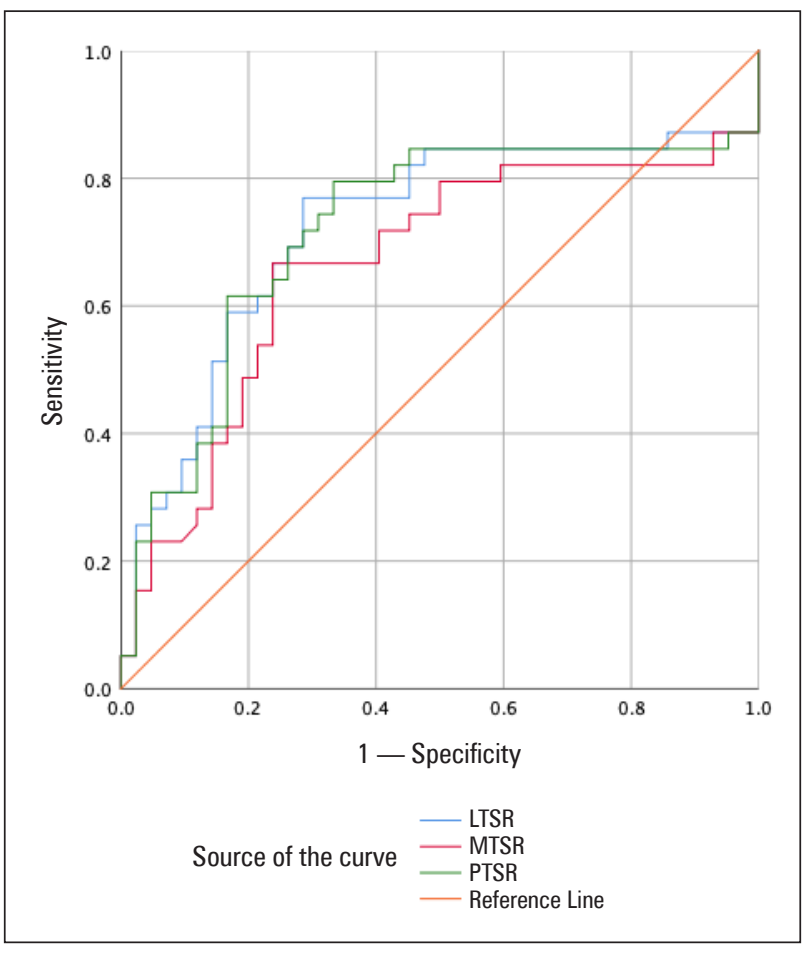

Figure 3. Receiver operating characteristic (ROC) curve analysis of the ratios of inflammatory cells to steroid receptor RNA activator (SRA) for metabolic syndrome (MetS) diagnosis. The accuracy of lymphocyte to SRA ratio (LTSR), monocyte to SRA (MTSR), and PBMCs to SRA ratio (PTSR) were 0.722 (95\% CI: 0.603-0.841, $p<0.001), 0.664$ (95\% CI: 0.539-0.789, $p=0.011$ ), and 0.718 (95\% CI: $0.598-0.838, p<0.001)$, respectively. The sensitivity and specificity of LTSR were $76.9 \%$ and $71.4 \%$, respectively. The sensitivity and specificity of MTSR were $66.7 \%$ and $76.2 \%$, respectively. The sensitivity and specificity of PTSR were 79.5\% and $66.7 \%$, respectively

coactivates GR as part of a ribonucleoprotein complex with p160 coactivators. In addition, SRA promotes GR-mediated transactivation in a ligand-dependent manner [4]. It was proven by Shannon Liu et al. that SRA gene knock-out could protect against obesity and improves glucose tolerance in high-fat-diet mice. In their study, they postulated that it might be associated with PPAR $\gamma$ [17]. SRA promotes adipogenesis and regulates insulin sensitivity in mouse adipocytes not only by acting as coactivator for PPAR $\gamma$, but also via multiple additional mechanisms. For example, SRA can promote S-phase entry during the mitotic clonal expansion phase of adipogenesis. It can also regulate cell cycle gene expression and suppress the expression of adipocyte-related inflammatory genes. What is more, SRA can inhibit TNF- $\alpha$-induced phosphorylation of c-Jun NH2-termianl kinase, which is implicated in the development of insulin resistance in mouse adipose tissue [18]. Several studies have found that SRA could regulate many inflammatory response genes such as zinc finger protein 36 , toll-like receptor 4 , haptoglobin, and 
$\mathrm{MCP}-1$. The most strongly regulated gene in this group is MCP-1, which is a macrophage-attracting chemokine highly expressed in adipose tissue. Overexpression of MCP-1 has been shown to contribute to macrophage infiltration of adipose tissue and insulin resistance. SRA may have anti-inflammatory functions within adipose tissue, which might contribute to the ability of SRA to enhance insulin sensitivity [19].

As a window, PBMCs (mostly consisting of lymphocytes) can convert psychosocial stress into cellular dysfunction and eventually contribute to the pathophysiology of lifestyle-related diseases such as DM, CVD, and atherosclerosis [11]. Chen et al. conducted a cross-sectional study on 852 participants $(n=598$ with MetS and $n=254$ without MetS) in southern China. They found that the severity of MetS was significantly positively associated with the WBC count, neutrophil count, and total lymphocyte count [20]. In the present study, we found that LTSR, MTSR, and PTSR can be good indicators for diagnosing MetS. It is the first time that we have investigated the relationship between SRA in PBMCs and the inflammatory cells to SRA ratios and $\mathrm{MetS}$ at a population level. The reasonable diagnostic accuracy of SRA and the ratios above indicate their clinical value in the diagnosis of MetS. However, well-designed prospective studies with larger sample sizes are required to validate these findings. Also, the expressions of SRA in circulation should also be detected, and the certain mechanism of SRA on GR and metabolic components need to be further identified in in vivo and in vitro levels.

\section{Conclusion}

Our findings suggest that SRA in PBMCs may play a vital role in MetS by targeting GR. What is more, the inflammatory cell to SRA ratios have demonstrated higher specificity and sensitivity in the diagnosis of MetS compared with SRA. These findings may unveil new targets for the prevention, diagnosis, and treatment of stress- and inflammation-related disorders including MetS.

\section{Funding}

This work was supported by the President Foundation of The Third Affiliated Hospital of Southern Medical University(No. YQ2021016), “Thirteenth Five-Year Plan" of Guangdong Province Educational Science (No. 2020GXJK441), the Project of Traditional Chinese Medicine Bureau of Guangdong Province (CN) (No. 20191228 to ZY), Science and Technology Planning Project of Huadu District, Guangzhou City (No. 21-HDWS-059).

\section{References}

1. Grundy SM. Metabolic syndrome pandemic. Arterioscler Thromb Vasc Biol. 2008; 28(4): 629-636, doi: 10.1161/ATVBAHA.107.151092, indexed in Pubmed: 18174459.

2. Sookoian S, Pirola CJ. Metabolic syndrome: from the genetics to the pathophysiology. Curr Hypertens Rep. 2011; 13(2): 149-157, doi: 10.1007/s11906-010-0164-9, indexed in Pubmed: 20957457.

3. Lu J, Wang L, Li M, et al. 2010 China Noncommunicable Disease Surveillance Group. Metabolic Syndrome Among Adults in China: The 2010 China Noncommunicable Disease Surveillance. J Clin Endocrinol Metab. 2017; 102(2): 507-515, doi: 10.1210/jc.2016-2477, indexed in Pubmed: 27898293.

4. Juster RP, McEwen BS, Lupien SJ. Allostatic load biomarkers of chronic stress and impact on health and cognition. Neurosci Biobehav Rev. 2010; 35(1): 2-16, doi: 10.1016/j.neubiorev.2009.10.002, indexed in Pubmed: 19822172 .

5. Wang M. The role of glucocorticoid action in the pathophysiology of the Metabolic Syndrome. Nutr Metab (Lond). 2005; 2(1): 3 , doi: 10.1186/1743-7075-2-3, indexed in Pubmed: 15689240.

6. Lai XN, Li J, Tang LB, et al. MiRNAs and LncRNAs: Dual Roles in TGF- $\beta$ Signaling-Regulated Metastasis in Lung Cancer. Int J Mol Sci. 2020; 21(4), doi: 10.3390/ijms21041193, indexed in Pubmed: 32054031.

7. Lin W, Zhou Q, Wang CQ, et al. LncRNAs regulate metabolism in cancer. Int J Biol Sci. 2020; 16(7): 1194-1206, doi: 10.7150/ijbs.40769, indexed in Pubmed: 32174794

8. Sathishkumar C, Prabu P, Mohan V, et al. Linking a role of lncRNAs (long non-coding RNAs) with insulin resistance, accelerated senescence, and inflammation in patients with type 2 diabetes. Hum Genomics. 2018; 12(1): 41, doi: 10.1186/s40246-018-0173-3, indexed in Pubmed: 30139387.

9. Caldwell KK, Hafez A, Solomon E, et al. Arsenic exposure during embryonic development alters the expression of the long noncoding RNA growth arrest specific-5 (Gas5) in a sex-dependent manner. Neurotoxicol Teratol. 2018; 66: 102-112, doi: 10.1016/j.ntt.2017.11.003, indexed in Pubmed: 29132937.

10. Liu C, Wu HT, Zhu N, et al. Steroid receptor RNA activator: Biologic function and role in disease. Clin Chim Acta. 2016; 459: 137-146, doi: 10.1016/j. cca.2016.06.004, indexed in Pubmed: 27282881.

11. Wang SS, Li YQ, Liang YZ, et al. Expression of miR-18a and miR-34c in circulating monocytes associated with vulnerability to type 2 diabetes mellitus and insulin resistance. J Cell Mol Med. 2017; 21(12): 3372-3380, doi: 10.1111/jcmm.13240, indexed in Pubmed: 28661068.

12. Huang Y, Zhao L, Yan Y, et al. PBMCs to Stress-Associated miR-18a-5p and miR-22-3p Ratios as New Indicators of Metabolic Syndrome. Biomed Res Int. 2020; 2020: 8159342, doi: 10.1155/2020/8159342, indexed in Pubmed: 32382575.

13. Dong J, Liang YZ, Zhang J, et al. Potential Role of Lipometabolism-Related MicroRNAs in Peripheral Blood Mononuclear Cells as Biomarkers for Coronary Artery Disease. J Atheroscler Thromb. 2017; 24(4): 430-441, doi: 10.5551/jat.35923, indexed in Pubmed: 27629254.

14. Chatzopoulou A, Roy U, Meijer A, et al. Transcriptional and Metabolic Effects of Glucocorticoid Receptor $\alpha$ and $\beta$ Signaling in Zebrafish. Endocrinology. 2015; 156(5): 1757-1769, doi: 10.1210/en.2014-1941, indexed in Pubmed: 25756310.

15. Whorwood CB, Donovan SJ, Flanagan D, et al. Increased glucocorticoid receptor expression in human skeletal muscle cells may contribute to the pathogenesis of the metabolic syndrome. Diabetes. 2002; 51(4): 1066-1075, doi: 10.2337/diabetes.51.4.1066, indexed in Pubmed: 11916927.

16. Michailidou Z, Jensen MD, Dumesic DA, et al. Omental 11beta-hydroxysteroid dehydrogenase 1 correlates with fat cell size independently of obesity. Obesity (Silver Spring). 2007; 15(5): 1155-1163, doi: 10.1038/oby.2007.618, indexed in Pubmed: 17495191.

17. Liu S, Sheng L, Miao H, et al. SRA gene knockout protects against diet-induced obesity and improves glucose tolerance. J Biol Chem. 2014; 289(19): 13000-13009, doi: 10.1074/jbc.M114.564658, indexed in Pubmed: 24675075.

18. Xu B, Gerin I, Miao H, et al. Multiple roles for the non-coding RNA SRA in regulation of adipogenesis and insulin sensitivity. PLoS One. 2010; 5(12): e14199, doi: 10.1371/journal.pone.0014199, indexed in Pubmed: 21152033.

19. Jiang $\mathrm{B}$, Wang $\mathrm{H}, \mathrm{Xu} \mathrm{H}$. Steroid receptor RNA activator affects the development of poststroke depression by regulating the peroxisome proliferator-activated receptor $\gamma$ signaling pathway. Neuroreport. 2020; 31(1): 48-56, doi: 10.1097/WNR.0000000000001367, indexed in Pubmed: 31714481.

20. Chen H, Xiong C, Shao X, et al. Lymphocyte To High-Density Lipoprotein Ratio As A New Indicator Of Inflammation And Metabolic Syndrome. Diabetes Metab Syndr Obes. 2019; 12: 2117-2123, doi: 10.2147/DMSO. S219363, indexed in Pubmed: 31686883. 